\title{
Neutral Sphingomyelinase 2 (SMPD3) Deficiency in Mice Causes Chondrodysplasia with Unimpaired Skeletal Mineralization
}

\author{
Wilhelm Stoffel, ${ }^{* \dagger \dagger}$ Ina Hammels, ${ }^{* \dagger}$ Britta Jenke, ${ }^{* \dagger}$ Inga Schmidt-Soltau, ${ }^{* \dagger}$ and Anja Niehoff
}

\begin{abstract}
From the Laboratory of Molecular Neurosciences, * Center of Biochemistry, Faculty of Medicine, the Center of Molecular Medicine, ${ }^{\dagger}$ the Cluster of Excellence, Cellular Stress Responses in Age Associated Diseases, ${ }^{\ddagger}$ and the Institute of Biomechanics and Orthopedics, ${ }^{\S}$ German Sport University Cologne, Cologne Center for Musculoskeletal Biomechanics, Faculty of Medicine, University of Cologne, Cologne, Germany
\end{abstract}

\author{
Accepted for publication \\ May 14, 2019. \\ Address correspondence to \\ Wilhelm Stoffel, M.D., Ph.D., \\ Center of Biochemistry, Uni- \\ versity of Cologne, Joseph- \\ Stelzmann-Str.52, 50931 Co- \\ logne, Germany. E-mail: \\ wilhelm.stoffel@uni-koeln.de.
}

\begin{abstract}
SMPD3 deficiency in the neutral sphingomyelinase $\left(\mathrm{Smpd3}^{--}\right)$mouse results in a novel form of juvenile dwarfism, suggesting smpd3 is a polygenetic determinant of body height. SMPD3 controls homeostasis of the sphingomyelin cycle in the Golgi compartment, essential for membrane remodeling, initiating multiform vesicle formation and transport in the Golgi secretory pathway. Using the unbiased Smpd $3^{\%}$ genetic model, this study shows that the perturbed Golgi secretory pathway of chondrocytes of the epiphyseal growth zone leads to dysproteostasis, skeletal growth inhibition, malformation, and chondrodysplasia, but showed unimpaired mineralization in primary and secondary enchondral ossification centers. This has been elaborated by biochemical analyses and immunohistochemistry of long bones of $\mathrm{Smpd}^{-/}$mice. A more precise definition of the microarchitecture and three-dimensional structure of the bone was shown by peripheral quantitative computed tomography, high-resolution microcomputed tomography, and less precisely by dual-energy X-ray absorptiometry for osteodensitometry. Ablation of the Smpd3 locus as part of a 980-kb deletion on chromosome 8 in the fro/fro mutant, generated by chemical mutagenesis, is held responsible for skeletal hypomineralization, osteoporosis, and multiple fractures of long bones, which are hallmarks of human osteogenesis imperfecta. The phenotype of the genetically unbiased Smpd $3^{-/}$mouse, described here, precludes the proposed role of Smpd3 as a candidate gene of human osteogenesis imperfecta, but suggests SMPD3 deficiency as the pathogenetic basis of a novel form of chondrodysplasia. (Am J Pathol 2019, 189: 1831-1845; https://doi.org/10.1016/j.ajpath.2019.05.008)
\end{abstract}

Acid and neutral sphingomyelinases (SMases; sphingomyelin phosphodiesterases, SMPDs), sphingomyelin (SM), and ceramides have been implicated in multiple cellular functions, including signaling pathways in growth, differentiation, and apoptosis. ${ }^{1}$ Acid sphingomyelinase (SMPD1) is localized in the lysosomal compartment, the main site of sphingolipid catabolism. nSMases form a family of sphingomyelin phosphodiesterases consisting of SMPD2 (nSMase1), ${ }^{2}$ SMPD3 (nSMase2), ${ }^{3}$ and SMPD5. ${ }^{4}$ SMPD2 is localized in the endoplasmic reticulum and SMPD3 resides in the Golgi compartment, both as integral membrane proteins. SMPD3 is the dominant representative of the nSMase family. SMPD5 is a bona fide mitochondrial nSMase. ${ }^{4}$ They show up as gene cluster in the neighbor-joining dendrogram ${ }^{5}$ of representative members of a superfamily of phosphohydrolases.

Understanding the molecular mechanisms underlying the systemic and cell-specific actions of SMPD3 required the meticulous dissection of the role of SMPD3 among the SMPDs, which contribute to total cellular SMase activity. Unbiased systemic null allelic mouse mutants $\operatorname{Smpd1,}{ }^{6,7} \operatorname{Smpd2,}{ }^{8}$ and $\operatorname{Smpd} 3^{9}$ have been instrumental in disentangling the enigmatic

Supported by the Centre of Molecular Medicine Cologne; the Cluster of Excellence; Cellular Stress Responses in Aging Associated Diseases; and Deutsche Forschungsgemeinschaft grants Sto32/50-1 (W.S.), NI 1083/3-1 (A.N.), and FOR2722 (A.N.).

Disclosures: None declared. 
mechanism of their systemic phenotypic responses. Ablation of Smpdl, lysosomal acid sphingomyelinase, preferentially expressed in cells of the reticulo-endothelial system of liver, bone marrow, spleen, lung, macrophages, and Purkinje cells of the cerebellum, causes sphingomyelin storage disease and human Niemann-Pick disease type A.

The neutral sphingomyelinase isozyme SMPD3 is localized in the endoplasmic reticulum. SMPD2 expression is marginal compared with SMPD3. The Smpd2 $2^{-/}$mutant develops an inconspicuous phenotype and systemic SMPD2 deficiency causes no SM storage. ${ }^{8}$ SMPD3 is expressed ubiquitously. The dominant expression of Smpd3 in the central nervous system is restricted mainly to neurons. Minor expression is observed in oligodendrocytes and astrocytes. High extraneuronal expression occurs in chondrocytes of the growth zones of the skeletal system. Subcellular localization and cellular functions of SMPD3 have been divergently discussed. ${ }^{1-3,8-12}$ Analysis of the phenotype of the Smpd $3^{--}$mouse showed the mechanism underlying a novel basic function of SMPD3 in the sphingomyelin cycle confined to the Golgi compartment. Primary chondrocytes in culture were instrumental in unveiling the mechanism underlying the absence of SMPD3 in the sphingomyelin cycle of the Golgi compartment: Golgi membrane budding, the initial step for multiform vesicle formation, transport, and secretion in the Golgi secretory pathway is impaired and leads to dysproteostasis and intracellular accumulation of extracellular matrix proteins (ECM) in the ERtubular system and Golgi complex. ${ }^{13}$ Perturbation of the Golgi secretory pathway in chondrocytes of the epiphyseal growth plate leads to inhibition of longitudinal growth, malformation of long bones, and chondrodysplasia. ${ }^{10}$

SMPD3 deficiency in hypothalamic neurosecretory neurons during the postnatal growth phase impedes the hypothalamus-pituitary growth axis and causes combined pituitary hormone deficiency-associated systemic hypoplasia. ${ }^{13}$

The comprehensive studies of the phenotype of the Smpd $^{-/}$mouse, ${ }^{3,9,11}$ generated by targeted ablation of the Smpd3 locus, critically differs from the phenotype of the fragilitas ossium (fro/fro) mouse mutant, a mutant mouse strain, which has been isolated from stock of mice that were chemically mutagenized randomly. ${ }^{14,15}$ The phenotype of the fro/fro mutant is characterized by a chromosomal deletion, which includes the smpd3 locus. The fro/fro mouse develops severe hypomineralization leading to multiple bone fractures, which are hallmarks of human lethal osteogenesis imperfecta and chronic respiratory obstructive symptoms. ${ }^{16}$ Therefore, fro/fro is regarded as a risk gene of osteogenesis imperfecta.

SMPD3 is restricted to the perturbation of the Golgi secretory pathway in chondrocytes, ECM protein secretion, transport, and extracellular apposition in the epiphyseal growth zone for longitudinal growth. ${ }^{13}$ Here, we show that SMPD3 deficiency leaves mineralization, the essential step during bone ossification, unaffected.

Biochemical, specific staining methods and immunohistochemical analyses of chondrocytes (ECM), osteoblasts, and osteoclast-specific marker proteins of long bones of juvenile and adult mice were combined with physical methods for quantifying bone architecture and bone parameters in wildtype (WT) and Smpd $3^{-/}$littermates. Mineralization during ossification was assessed by dual-energy X-ray absorptiometry. Bone architecture and bone structural parameters were examined by peripheral quantitative computed tomography (pQCT) and high-resolution microcomputed tomography $(\mu \mathrm{CT})$. Volumetric analysis and cortical and trabecular bone parameters were evaluated separately.

The cumulative data of these studies indicate similar cortical and trabecular bone mineralization in adult WT and $S m p d 3^{--}$mice. They preclude Smpd3 as a risk gene of osteogenesis imperfecta, but suggest its candidate role in a novel form of chondrodysplasia.

\section{Materials and Methods}

\section{Mouse Strains}

The Smpd $3^{--}$mouse strain was developed in the Laboratory of Molecular Neuroscience, Center of Biochemistry, Faculty of Medicine, University of Cologne after 10 back-crossings maintained on a C57B1/6 background. ${ }^{9}$ WT mice were obtained from heterozygous Smpd $3^{-/} \times \mathrm{C} 57 \mathrm{~B} 1 / 6$ crossings. Mice were genotyped by PCR analysis of tail DNA. Animal protocols followed the principles and practices outlined in the NIH Guide for the Care and Use of Laboratory Animals, ${ }^{17}$ and breeding and tests were performed with permission of the local authorities [Landesamt für Natur, Umwelt und Verbraucherschutz (LANUV NRW)]. The animal studies reported in this article followed the Animal Research: Reporting of In Vivo Experiments (ARRIVE) Guidelines. ${ }^{18}$ All animals were kept under specific pathogen-free conditions. The light/dark cycle was 12 hours/ 12 hours; the animals had free access to water and a regular diet. Cohorts of sex-, age-, and weight-matched WT and $\operatorname{Smpd} 3^{-/-}$mice were used in this study.

\section{Lipidome Analysis}

Total lipids were extracted and homogenized in an Ultraturrax (IKA Labortechnik, Staufen, Germany) in 10 volumes of chloroform/methanol (C/M) 2:1 (vol/vol) and re-extracted with $\mathrm{C} / \mathrm{M} \mathrm{1:1}(\mathrm{vol} / \mathrm{vol})$ and C/M 1:2 (vol/vol) for 1 hour each at $37^{\circ} \mathrm{C}$ under a stream of nitrogen. The combined extracts of total lipids were dissolved in C/M 2:1 (vol/vol), washed with $2 \mathrm{~mol} / \mathrm{L} \mathrm{KCl}$ and water, and taken to dryness in a stream of nitrogen. Phospholipids were separated in solvent system chloroform/ethanol/triethylamine/water 60/70/70/14 (vol/ $\mathrm{vol} / \mathrm{vol} / \mathrm{vol}$ ), sphingolipids were separated in: chloroform/ methanol/water 65/25/4 (vol/vol/vol) using highperformance thin layer chromatography (HPTLC) plates (Merck, Darmstadt, Germany). Bands were visualized by primuline fluorescence $(0.2 \%$ in $80 \%$ acetone). Lipid bands were collected on fritted glass filters and eluted with C/M 2:1 
A

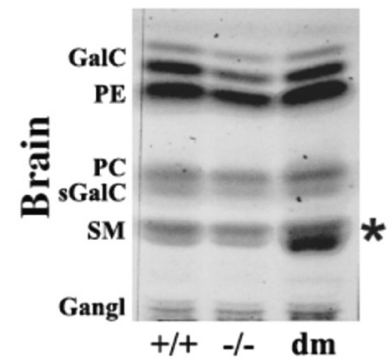

C

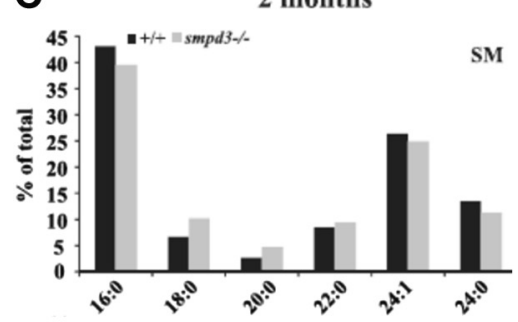

D

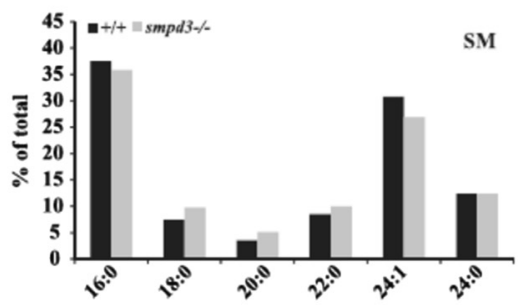

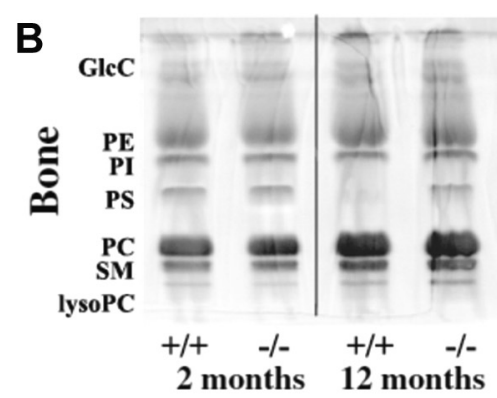

2 months

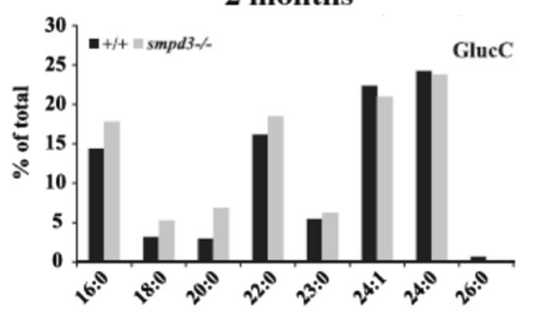

12 months

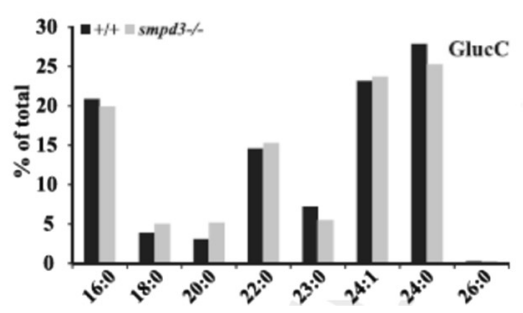

2 months
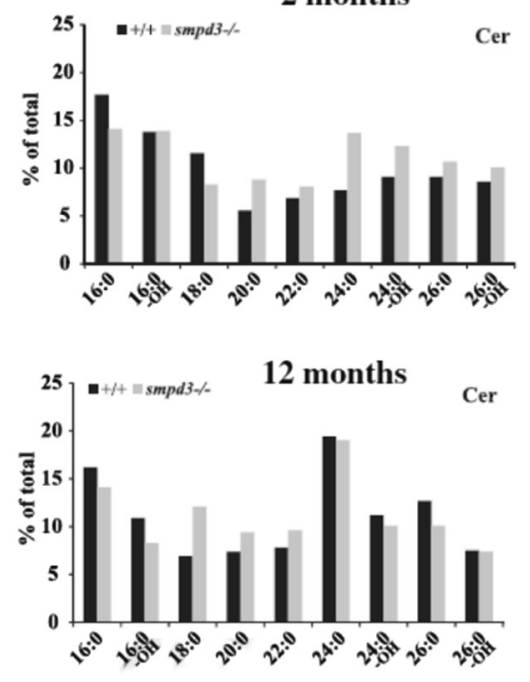

Figure 1 Smpd3 deficiency causes no sphingolipidosis. A: High-performance thin layer chromatography (HPTLC)-separation of brain lipid extracts of 4month-old wild-type (WT) $(+/+)$, Smpd $3^{\%}(-/-)$, and Smpd1 ${ }^{\%} /$ Smpd $3^{\%}(\mathrm{dm})$ mice. Solvent system: chloroform/methanol/water 65/25/4 (vol/vol/vol). Asterisk indicates SM storage in dm lane. B: HPTLC separation of bone lipid extracts of 2- and 12-month-old WT (+/+), Smpd3 $/-(-/-)$. Solvent system: chloroform/ethanol/triethylamine/water 60/70/70/14 ( $\mathrm{vol} / \mathrm{vol} / \mathrm{vol} / \mathrm{vol})$. C and D: Mass spectroscopic analysis (MS/MS) of bone neutral sphingolipids of 2month-old (C) and 12-month-old (D) WT and Smpd $\%$ mice. Cer, ceramides; GlucC, glucosylceramides; lysoPC, lyso-phosphatidylcholine; PC, phosphatidylcholine; PE, phosphatidylethanolamine; PI, phosphatidylinositol; PS, phosphatidylserine; sGalC, sulfo-Galactosylceramide; SM, sphingomyelin.

(vol/vol) into Sovirel tubes (SciLabware, Staffordshire, UK) and concentrated under $\mathrm{N}_{2}$ for analysis by mass spectrometry.

\section{Protein Analysis}

Freshly dissected bones of WT and $\operatorname{Smpd3^{-/}}$ male and female mice were homogenized mechanically in lysate buffer containing protease inhibitor cocktail (Complete; Roche, Penzberg, Germany). Protein concentrations were measured using the Pierce BCA protein assay kit (Thermo Fisher Scientific, Darmstadt, Germany). Protein aliquots $(100 \mu \mathrm{g})$ were separated by NuPAGE $4 \%$ to $12 \%$ BIS-TRIS gels and transferred to a nitrocellulose membrane, using the NuPAGE Western Blot system (Invitrogen, Darmstadt, Germany). Blots were immunostained overnight at $4^{\circ} \mathrm{C}$ with the following respective antibodies: anti-alkaline phosphatase (1:2000, ab108337, RRID:AB_10862036), anti-collagen I (1:500, ab21286, RRID:AB_446161), and anti-osteopontin (1:1000, ab181440) (all from Abcam, Cambridge, UK), and anti- $\alpha$ tubulin $(1: 12,000$, T6074, RRID:AB_477582; Sigma-Aldrich, St. Louis, MO). After washing, horseradish peroxidase-conjugated secondary antibodies were used and detected with the enhanced chemiluminescence system. Signals were quantified by densitometry using the ImageJ2 version 2.0.0-rc-3 (NIH, Bethesda, MD) program (RRID:SCR_003070).

\section{Histology and Immunohistochemistry}

Freshly prepared long bones (humerus) of male and female WT and Smpd $3^{-/}$mice, 2 and 12 months of age, were fixed in $4 \%$ buffered paraformaldehyde for 48 hours and were decalcified in 10\% EDTA/Tris pH 7 for 10 to 14 days with shaking. The buffer was exchanged once and the bones were transferred into $70 \%$ ethanol for paraffin embedding and processing for light and immunofluorescence microscopy. Sections $(5 \mu \mathrm{m})$ were permeabilized with $0.5 \%$ Triton X-100 (Sigma-Aldrich)/phosphate-buffered saline at $4^{\circ} \mathrm{C}$, and hematoxylin and eosin, Masson-Goldner, van Gieson, Alcian Blue, and von Kossa stained for transmission microscopy, following established protocols. For immunostaining, sections were blocked with $3 \%$ bovine serum albumin/ 

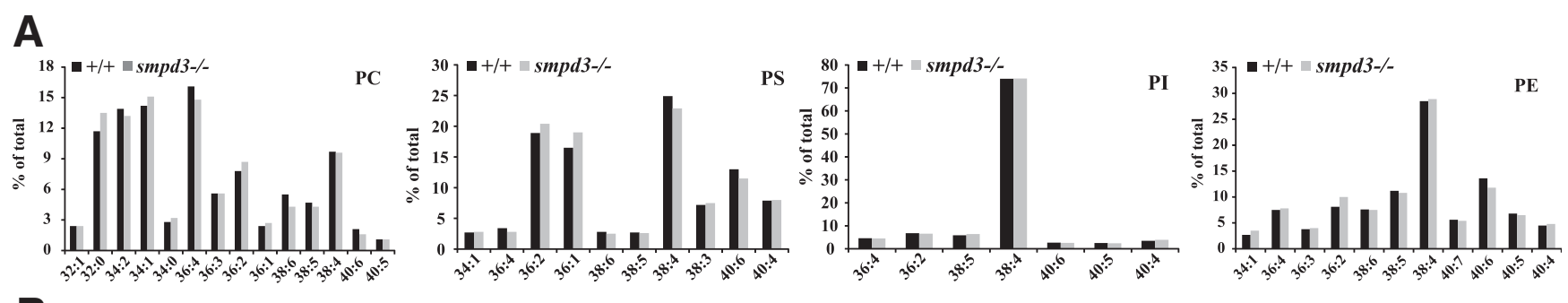

B
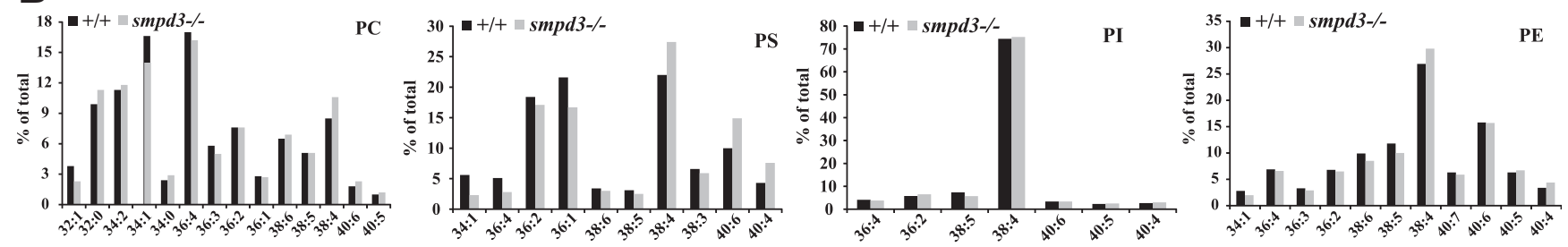

Figure 2 Bone phospholipidome of wild-type (WT) and Smpd $\%$ mice. MS/MS analysis of phospholipid classes of phospholipidomes of long bones (femora) of 2-month-old (A) and 12-month-old (B) WT and Smpd3\% mice, separated by high-performance thin layer chromatography (HPTLC). Solvent system: chloroform/ ethanol/triethylamine/water 60/70/70/14 (vol/vol/vol/vol). PC, phosphatidylcholine; PE, phosphatidylethanolamine; PI, phosphatidylinositol; PS, phosphatidylserine.

phosphate-buffered saline and $0.1 \%$ Triton X-100/phosphatebuffered saline and treated with respective antibody dilutions in phosphate-buffered saline at $4{ }^{\circ} \mathrm{C}$ overnight. The following antibodies and dilutions were used: anti-alkaline phosphatase (1:200, ab108337, RRID:AB_10862036), anti-collagen I (1:200, ab21286, RRID:AB_446161), and anti-osteopontin from Abcam (1:50, ab181440), and anti- $\alpha$ tubulin from Sigma-Aldrich (1:1000, T6074, RRID:AB_477582) and Cyanine dye 3 (Cy3)-labeled secondary antibody were used for immunofluorescence microscopy.

\section{Preparation of 0steoblasts}

Isolation and culture of osteoblastic cells of WT and Smpd $3^{-/}$mice, 2 and 12 months of age, was performed using an established procedure. ${ }^{5}$

\section{Microscopy}

The Slidescanner (SCN400; Leica, Wetzlar, Germany) and the software Aperio ImageScope version 12.2.2.5015 (RRID:SCR_014311), the Axio ImagerM1 microscope (Zeiss, Oberkochen, Germany), Imaris Software version 5.5 (RRID:SCR_007370), and AxioVision Imaging Software version 4.8.2.0 (RRID:SCR_002677; AxioVision, Oberkochen, Germany) were used. The TCS SP8X confocal microscope (Leica Microsystems), equipped with a PL Apo $63 \times / 1.40$ Oil CS2 objective, white-light laser (NKT Photonics, Regensdorf, Switzerland), and HyD detectors (Leica) were used for confocal microscopy.

\section{Quantitative X-Ray Absorptiometry}

Mineralization of skeletons of WT and Smpd $3^{-/}$littermates was measured by quantitative dual-energy X-ray absorptiometry. Anesthetized WT and $\operatorname{Smpd} 3^{-/-}$littermates were examined using a bench X-ray unit (HP Cabinet X-ray System-Faxitron series, model 43855A; Hewlett-Packard, McMinnville, OR), with single-side emulsion film (AgfaTs Structurix D4DW, NDT System; Grosche, Bottrop, Germany) at $40 \mathrm{kV}$ with exposure times of 25 seconds for young mice and $50 \mathrm{kV}$ and 48 seconds for adult mice.

\section{pQCT}

Right femora of 10- and 27-week-old female WT and Smpd $3^{-1-}$ mice were scanned by pQCT using the XCT Research M scanner and Stratec software version 5.50 (Stratec Medizintechnik GmbH, Pforzheim, Germany). For the measurements, isolated bones were placed, with the anterior surface upward, in a syringe filled with saline solution. After scout view, sections were made at the distal femoral metaphysis (at 15\%,17.5\%, and $20 \%$ of total bone length measured from the distal joint line) and at the midshaft (at $50 \%$ of total bone length). The voxel size was $500 \times 70 \times 70 \mathrm{~m}$. Each slice was analyzed by contour mode 1 , peel mode $20(30 \%)$, and cortical mode $1\left(710 \mathrm{mg} / \mathrm{cm}^{3}\right)$. At the femoral metaphysis, total cross-sectional bone area (crosssectional bone area, $\mathrm{mm}^{2}$ ), total bone mineral density (BMD, $\mathrm{mg} / \mathrm{cm}^{3}$ ), total bone mineral content (in $\mathrm{mg}$ ), trabecular crosssectional bone area (in $\mathrm{mm}^{2}$ ), trabecular bone mineral content (in $\mathrm{mg} / \mathrm{cm}^{3}$ ), and trabecular bone mineral content (in $\mathrm{mg}$ ) were determined as the mean of three slices. At the mid-diaphysis, the cortical area (in $\mathrm{mm}^{2}$ ), the cortical bone mineral density (in $\mathrm{mg} /$ $\mathrm{cm}^{3}$ ), the cortical bone mineral content (in $\mathrm{mg}$ ), the cortical thickness (in $\mathrm{mm}$ ), the periosteal circumference ( $\mathrm{mm}$ ), and the endosteal circumference $(\mathrm{mm})$ were evaluated. Reproducibility of pQCT measurements with the settings described here was determined by repeated scans of mouse femora with repositioning. The root-mean square average coefficient of variation (CV\%) values were $2.2 \%$ for trabecular bone mineral density and $0.7 \%$ for cortical bone mineral density. 
A
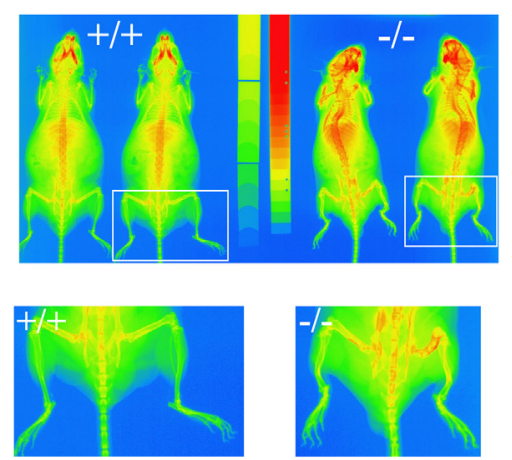

C

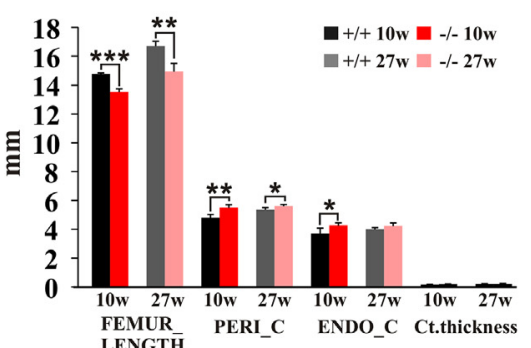
LENGTH

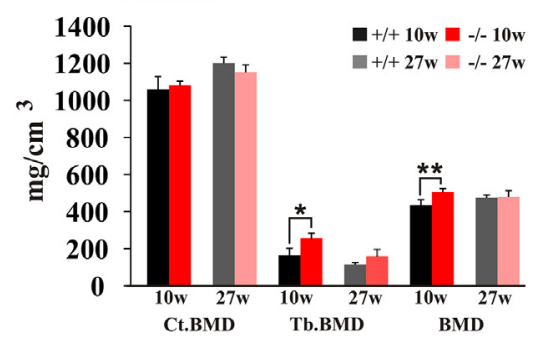

High-Resolution $\mu \mathrm{CT}$

Bone microstructure of right femora from 10- and 27week-old female WT and $S m p d 3^{-/}$mice was analyzed using a high-resolution $\mu \mathrm{CT}$. The scanner $\mu \mathrm{CT} 35$ (Scanco Medical AG, Bassersdorf, Switzerland) was used for image acquisition. Following the guidelines for assessment of bone microstructure in rodents using $\mu \mathrm{CT},{ }^{5}$ dissected femora were scanned in saline with an isotropic voxel size of $7 \mu \mathrm{m}, 70-\mathrm{kV}$ $\mathrm{X}$-ray tube voltage, 114-mA tube current, and 400-ms integration time. The volume of interest of trabecular bone was evaluated in the region $0.75-\mathrm{mm}$ underneath the growth plate (142 slices, $1 \mathrm{~mm}$ ) and of cortical bone in the midshaft (107 slices, $0.75 \mathrm{~mm}$ ). Segmentation steps were applied with support $=1$, sigma $=0.8$ at the metaphysis and with support $=2$, sigma $=1.2$ at the diaphysis. Bone tissue was segmented using a global thresholding algorithm $(22.5 \%$ of the maximum gray scale value for the metaphysis, $32 \%$ for the diaphysis). To remove image noise, gray-scale data of the raw CT images were processed using a 3-dimensional Gaussian filter algorithm. ${ }^{19}$ Cortical parameters included bone tissue area (in $\mathrm{mm}^{2}$ ), cortical bone area (in $\mathrm{mm}^{2}$ ), marrow area $\left(\mathrm{mm}^{2}\right)$, cortical thickness (in $\mathrm{mm}$ ), cortical area fraction (cortical bone area/tissue area, \%), and cortical bone mineral density (in mg hydroxyapatite $/ \mathrm{cm}^{3}$ ). At the metaphysis bone volume (in $\mathrm{mm}^{3}$ ), tissue volume (in $\mathrm{mm}^{3}$ ), bone volume
Figure 3 Regular mineralization of adult Smpd $3^{\%}$ mice. A and B: Images of quantitative radiography-densitometry of wild-type (WT) $(+/+)$ and $\mathrm{Smpd}^{-/-}(-/-)$anterior-posterior (ap) (A) and lateral whole body position (B). The boxed areas from panel $\mathbf{A}$ (ap pelvis/hind legs) and the boxed areas in panel $\mathbf{B}$ (lateral fore legs) are enlarged in the corresponding panels below. C: Peripheral quantitative computed tomography analysis from female femora of WT and Smpd $3^{-/}$mice. Periosteal circumference (PERI_C), endosteal circumference (ENDO_C), cortical bone thickness (Ct.thickness), cortical bone mineral content (Ct.BMC), trabecular bone mineral content (Tb.BMC), total bone mineral content (BMC), cortical bone density (Ct.BMD), trabecular bone density (Tb.BMD), total bone density (BMD), cortical cross-sectional bone area (Ct.CSA), trabecular cross-sectional bone area (Tb.CSA), and total cross-sectional bone area (CSA) are shown. $n=5$ per age and genotype. ${ }^{*} P<0.05,{ }^{* *} P<0.01$, and ${ }^{* *} P<0.001$. w, weeks. fraction (bone volume/tissue volume, \%), trabecular separation (in $\mathrm{mm}$ ), trabecular thickness (in $\mathrm{mm}$ ), trabecular number (in $1 / \mathrm{mm})$, connectivity density $\left(1 / \mathrm{mm}^{3}\right)$, and trabecular bone mineral density (in $\mathrm{mg}$ hydroxyapatite $/ \mathrm{cm}^{3}$ ) were determined.

\section{Real-Time PCR}

RNA was isolated from WT and Smpd $3^{--}$male and female femora of littermates using TRIzol (Invitrogen). Ten microgram of total RNA was reverse-transcribed using a SuperscriptTMII Reverse Transcriptase kit (Thermo Fisher Scientific). The following primer pairs were used in quantitative PCR reactions: osteopontin forward: 5'-CCCGGTGAAAGTGACTGATT-3', osteopontin reverse: 5'-CCATCGTCATCATCATCGTC-3'; osteocalcin forward: 5'-GCGCTCTGTCTCTCTGACCT-3', osteocalcin reverse: $5^{\prime}$-TTTGTAGGCGGTCTTCAAGC-3'; alkaline phosphatase forward: 5'-GCTGATCATTCCCACGTTTT- $3^{\prime}$, alkaline phosphatase reverse: $5^{\prime}$-CTGGGCCTGGTA-GTTGTTGT-3'; bone morphogenic factor 2 forward: 5'-TGCTAACGACACCCGCAGCCCTCCACAACC-3', bone morphogenic factor 2 reverse: 5'-CAAGCCAAACACAAACAGCGGAAGCGCCTC $-3^{\prime}$; col2A forward: 5'-CCACTTCAGC-TATGGC-3', col2A reverse: 5'-CGGTACTCGATGACGG-3'; comp forward: 5'-GAGCAGACGTACTGGC-3', comp reverse: 5'-GGGAGAAGCAGAAGACA -3'; and hgprt forward: 5'-GCTGACCTGCTGGATTACATTAAAGCACTG-3', hgprt 
reverse: 5'-ATTCCTGAAGTACTCATTATAGTCAAGGGC$3^{\prime}$. Hgprt was used as an internal standard. Quantitative PCR reactions were performed with the Prism 7900HT (ABI, Darmstadt, Germany) using a 96-well format, the Fast SYBR Green Master Mix (Applied Biosystems, Darmstadt, Germany), following the manufacturer's protocol. Data were analyzed using the comparative $2[$-delta delta $\mathrm{C}(\mathrm{T})]$ method (2- $\Delta \Delta \mathrm{Ct}$ method).

\section{Statistical Analysis}

Results are expressed as means \pm SEM. Statistical analysis of differences between individual experimental groups was performed using QuickCalcs (GraphPad, San Diego, CA). An unpaired two-tailed $t$-test was used. $P$ values of $\leq 0.05$ were considered significant.
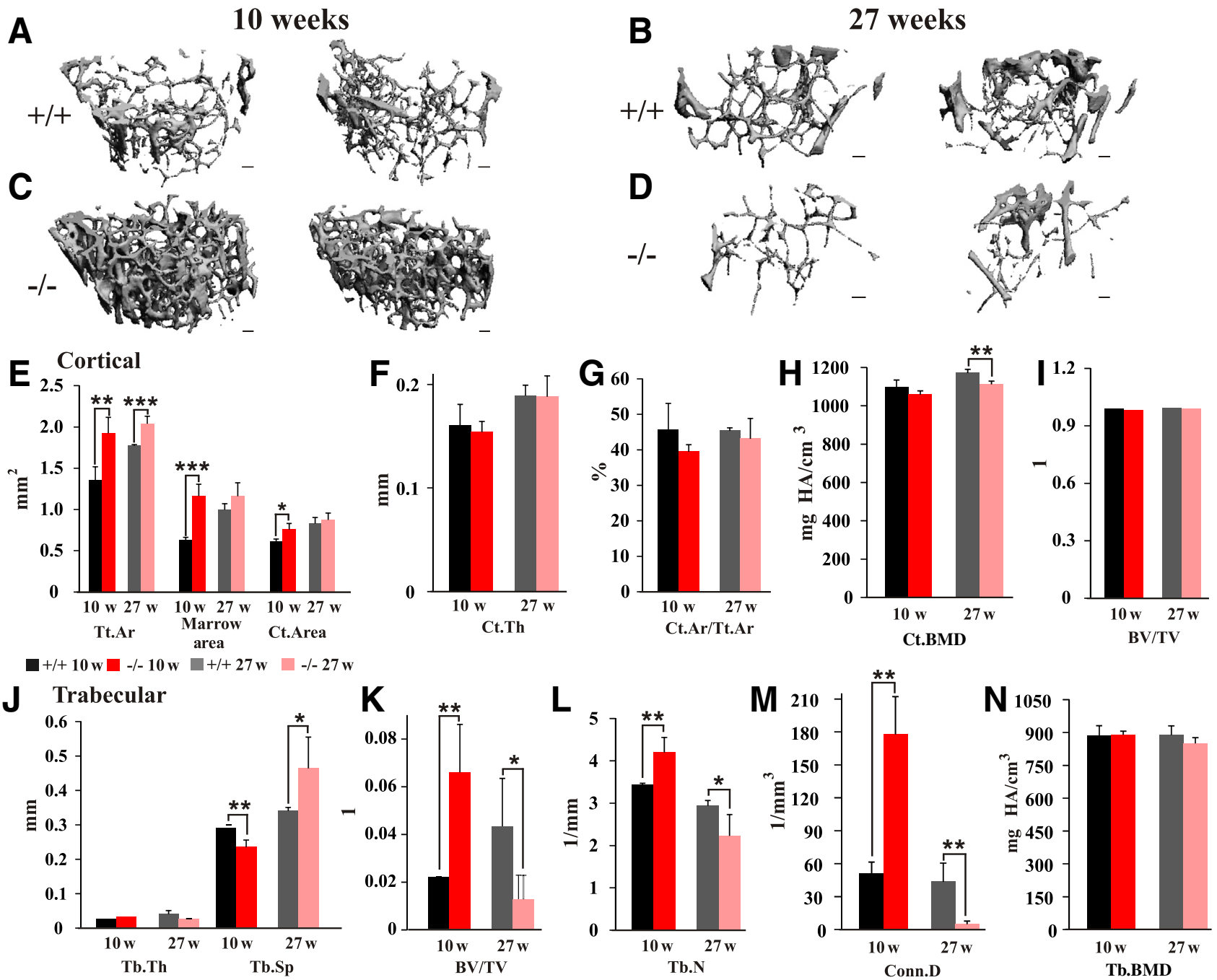

Figure 4 Microcomputed tomography of trabecular and cortical bone. A-D: Three-dimensional reconstructed trabecular architecture of femora of 10week-old (A) and 27-week-old (C) wild-type (WT) mice and 10-week-old (B) and 27-week-old (D) Smpd3\%- mice. E-N: Microstructural cortical (E-I) and trabecular $(\mathbf{J}-\mathbf{N})$ architecture. Trabecular bone was evaluated in the region $0.75 \mathrm{~mm}$ underneath the growth plate (142 slices, $1 \mathrm{~mm})$. Cortical bone was analyzed in the midshaft (107 slices, $0.75 \mathrm{~mm}) .{ }^{17}$ Total bone area (Tt.Ar), marrow area, cortical bone area (Ct.Area), cortical thickness (Ct.Th), bone area fraction (Ct.Ar/Tt.Ar), cortical bone mineral density (Ct.BMD), relative bone volume (BV/TV), trabecular thickness (Tb.Th), trabecular separation (Tb.Sp), trabecular number (Tb.N), connectivity density (Conn.D), and trabecular bone mineral density (Tb.BMD) are shown. $n=5$ per age and genotype. ${ }^{*} P<0.05,{ }^{* *} P<0.01$, and ${ }^{* * *} P<0.001$. Scale bars: $100 \mu \mathrm{m}(\mathbf{A}-\mathbf{D})$. HA, hydroxyapatite; w, weeks. 

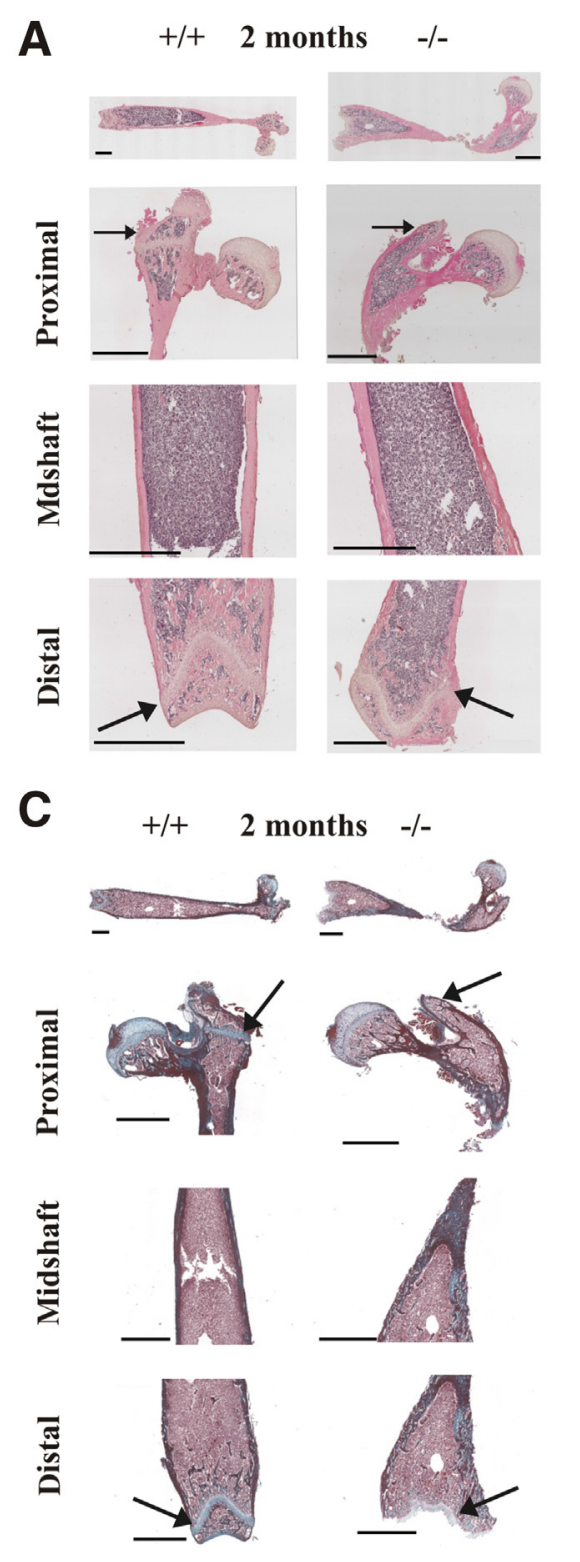

B

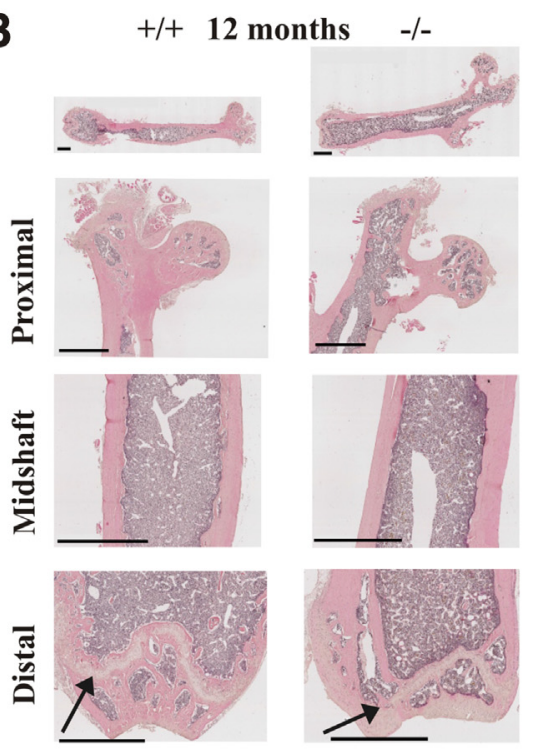

D
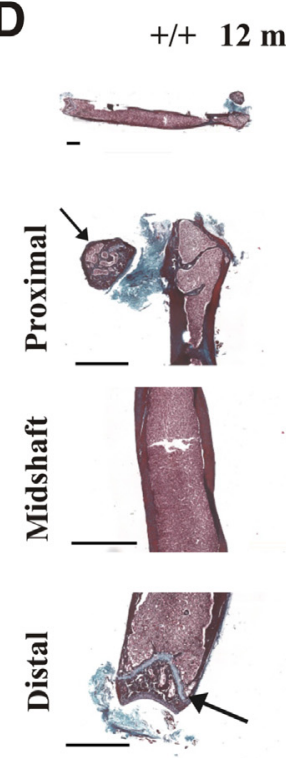

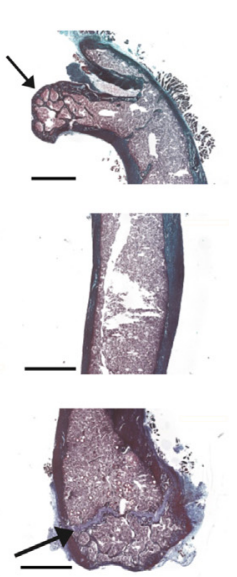

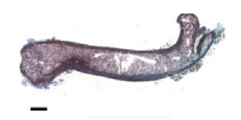

Figure 5 Morphology of growth and ossification zones and cortices of 2-month-old and 12-month -old wild-type (WT) and Smpd $3^{\%}$ mice femora. Longitudinal sections of femora from 2-month-old (A) and 12-month-old (B) WT (+/+) and Smpd3 $3^{--}$ $(-/-)$, stained with hematoxylin and eosin, and 2month-old (C) and 12-month-old (D) $+/+$ and -/- stained according to Masson-Goldner (red, cytoplasm and fibrils; blue/green, collagen, acid mucopolysaccharides, and bones). Arrows indicate growth zones. Scale bars $=1 \mathrm{~mm}$. with high Smpd3 expression. Combined HPTLC separation and quantitative analysis by mass spectrometry (MS)/MS showed highly similar concentrations of phospholipidome and sphingolipidome classes (Figures 1 and 2).

Smpd3 ablation left SM concentration of bone and brain of $S m p d 3^{-/}$mice unaffected (Figure 1, A and B). Introduction of the $S m p d 1^{-\alpha}$ locus into the Smpd3 $3^{-\alpha}$ genome, however, induced systemic sphingomyelin storage, shown in the HPTLC of brain of the Smpd1 $1^{-/} / S m p d 3^{-/}$double mutant (Figure 1A). Sphingomyelin, ceramides, and glucosylceramide species and concentration in total lipid extract of bones of 2-month-old (Figure 1C) and 12-month-old (Figure 1D) WT and $\operatorname{Smpd} 3^{-/}$mice showed no differences in MS/MS analysis.

The species of phospholipid classes in the lipidome of bones of 2- and 12-month-old WT and Smpd $3^{-/-}$mice were similar in the MS/MS analysis (Figure 2).
Parameters of mineralization of the cortical and trabecular architecture of long bones of cohorts of 10- and 27-weekold WT and Smpd 3 male mice were characterized pQCT for 3-dimensional volumetric quantitative bone morphometry and $\mu \mathrm{CT}$.

Quantification of X-radiography suggested equal mineralization during ossification of the skeleton of 12-month-old WT and $\operatorname{Smpd}^{-/}$mice. Images of dual-energy X-ray absorptiometry of ap- and lateral-positioned 12-month-old $S m p d 3^{-/}$mice showed the severe malformation of long bones and joints, which developed independently of ongoing mineralization (Figure 3, A and B). Densitometry indicated an even higher mineralization of the pelvis, femur, and tibia and fibula of hind legs in $\operatorname{Smpd} 3^{-/}$mice.

In addition, pQCT parameters of cortical and trabecular mineralization of $\mathrm{Smpd}^{-/-}$long bones significantly exceeded those of WT mice (Figure $3 \mathrm{C}$ ). The length of $S m p d 3^{--}$femora 
A
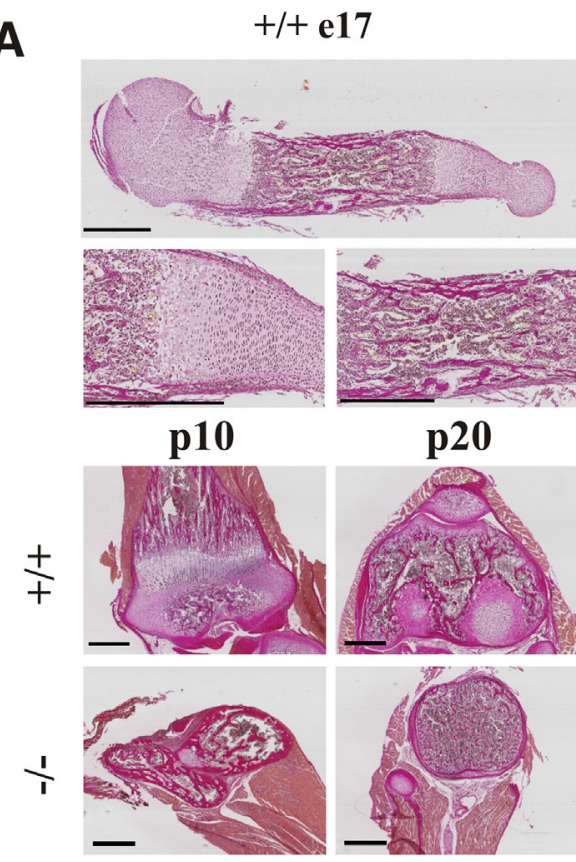

B
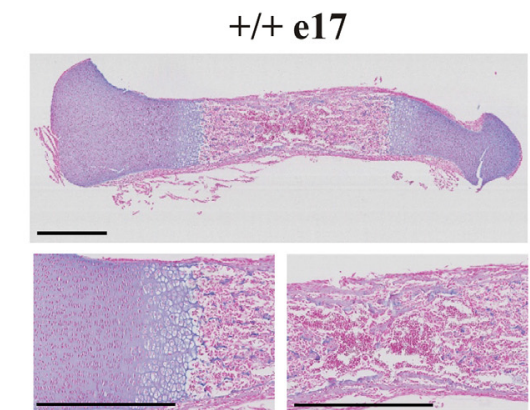

p10
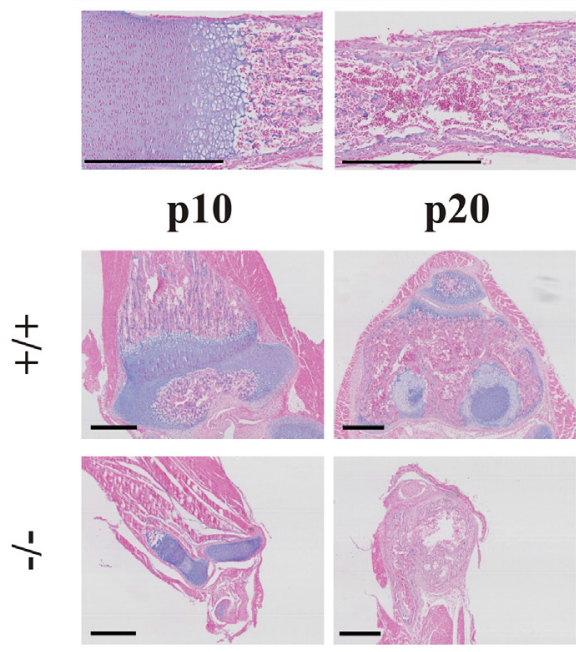

p20
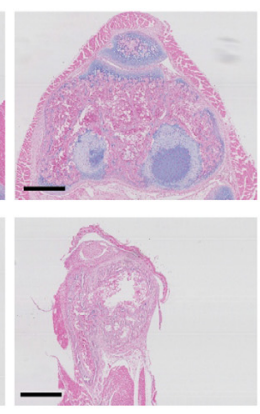
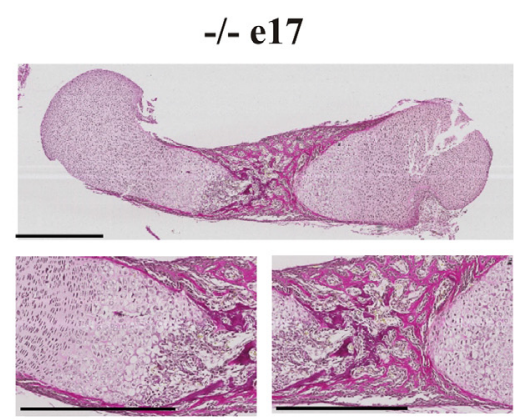

2 months
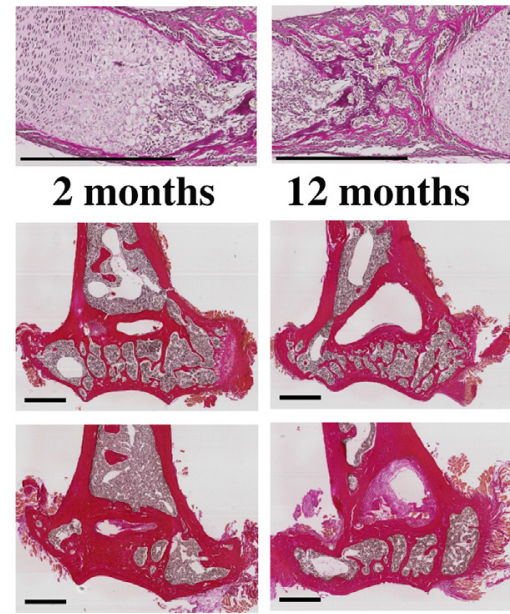

12 months
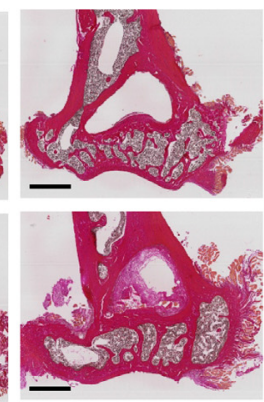

$-/-\mathbf{e} 17$
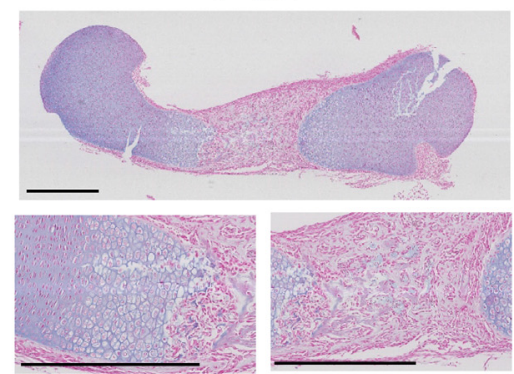

2 months

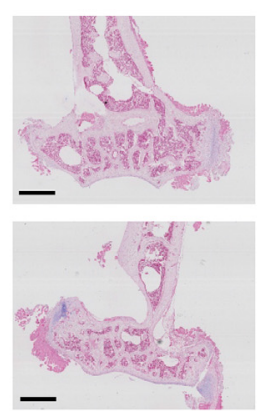

Figure 6 Histology of extracellular matrix and cartilage in growth zones of long bones of wild-type (WT) and Smpd3 $3^{-/}$mice. Van Gieson staining collagen (connective tissue; $\mathbf{A}$ ) and Alcian blue glycosaminoglycans (cartilage; B) staining of e17, p10, p20, 2-month-old, and 12-month-old WT $+/+$ and $S m p d 3^{--}$mice. Scale bars $=500 \mu \mathrm{m}$. e, embryonal day; $p$, postnatal day.

remained approximately $15 \%$ to $20 \%$ shorter in $\mathrm{Smpd}^{-/}$than in WT cohorts throughout their life span (Figure 3C).

\section{High-Resolution $\mu \mathrm{CT}$ Analysis of Cortical and} Trabecular Structure in Smpd3\% $\%$ Bones

Microstructural cortical and trabecular architectures of femora of both 10- and 27-week-old WT and Smpd3 $3^{-1-}$ mice were analyzed using 3-dimensional high-resolution $\mu \mathrm{CT}$. Dissected femora were scanned at the metaphysis and at the diaphysis.

Cortical parameters derived from $\mu \mathrm{CT}$ analysis of femora of female WT and Smpd $3^{-/}$mice indicated similar cortical thickness, cortical bone area fractions (cortical bone area/tissue area), cortical total mineralized bone, and relative bone volume (bone volume/tissue volume) (Figure 4, F-I). The total bone area was significantly higher in $\operatorname{Smpd} 3^{-/-}$mice, marrow area, and cortical bone area in 10-week-old Smpd $3^{-/}$mice (Figure 4E). 

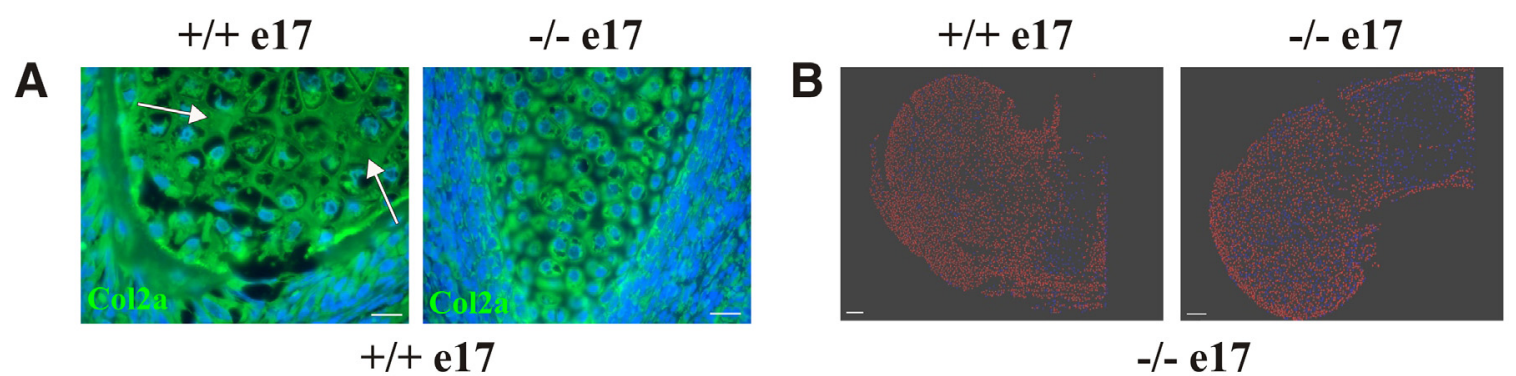

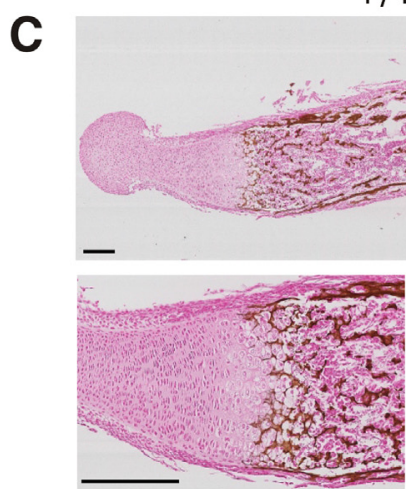
$+/+\mathbf{p 1 0}$

E
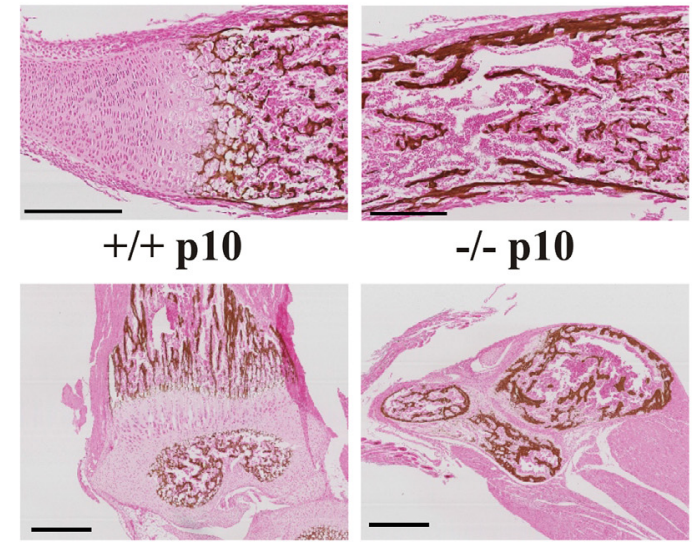

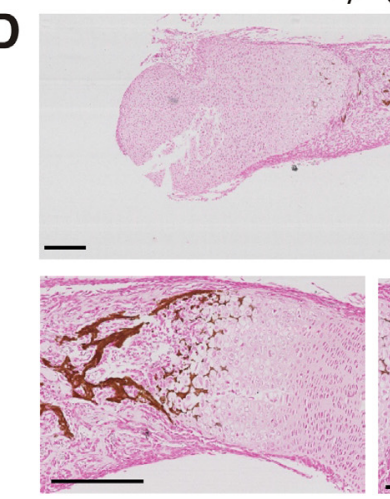

$+/+\mathbf{p 2 0}$

F

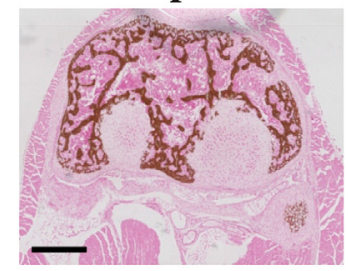

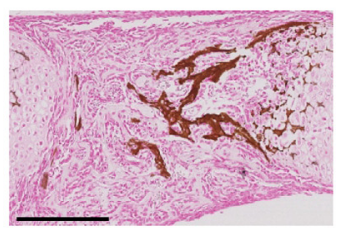

$-/-$ p20

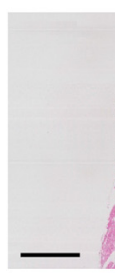

Figure 7 Extracellular matrix development and cell proliferation in growth zones of femora of e17, p10, p20 old wild-type (WT) $+/+$ and Smpd3 $\%$ mice. A and B: Immunohistochemistry using anti collagen $2 \mathrm{~A}$ (Col2A) (arrows indicate abundant extracellular matrix production in control humerus). C-F: von Kossa staining monitors mineralization in e17 (C and D), p10 (E), and p20 (F) +/+ and Smpd3 $\%$ femora. Scale bars: $20 \mu \mathrm{m}(\mathbf{A}) ; 100 \mu \mathrm{m}(\mathbf{B}) ; 250 \mu \mathrm{m}(\mathbf{C}$ and D) , 500 $\mu \mathrm{m}$ (E and $\mathbf{F})$. e, embryonal day; p, postnatal day.

Trabecular bone was evaluated in the region underneath the growth plate (metaphysis). The trabecular number, connectivity density, and relative bone volume (bone volume/tissue volume) was significantly higher in juvenile, but reduced in adult, $\mathrm{Smpd}^{-/-}$mice (Figure $5 \mathrm{~K}, \mathrm{~L}$, and N), which is reflected in the distance between trabeculae (trabecular separation) (Figure 4J). Images of the 3dimensional reconstructed trabecular architecture of femora from 10- and 27-week-old female WT and $\mathrm{Smpd}^{-/}$mice confirmed the quantitative data of the trabecular parameters (Figure 4, A-D). Trabecular and cortical total mineralized bone of WT and $S m p d 3^{-/}$mice were unchanged (Figure 4, H and N). Relative bone volume, trabecular number, and connectivity density are elevated in 10-week-old, but reduced at 27-week-old female Smpd3 mice (Figure 4, K, L, M).

Skeletal Growth Inhibition, Malformation, and Chondrodysplasia of Joints and Regular Mineralization of Bones

Hematoxylin and eosin and Masson-von Goldner staining of longitudinal sections of $\operatorname{Smpd} 3^{-/}$femora showed severe malformation of the short femora (Figure 5). Spatially disordered zones of resting, proliferative, pretrophic, and hypertrophic chondrocytes in growth plates of the proximal and distal epiphyses and the articular cartilage, associated with perturbed invasion of osteoblasts, bone marrow cells, blood vessels, and osteoclasts, involved in bone remodeling and removal of cartilage matrix at the perturbed ossification front, characterize the morphology of the growth plate of Smpd $3^{-/}$long bones. The cortex of midshaft femora of WT and Smpd $3^{--}$of 2- and 12-month-old mice showed no difference in thickness, in support of the quantitative radiography/densitometry, $\mathrm{pQCT}$, and $\mu \mathrm{CT}$ data. No fractures in the skeleton of $\operatorname{Smpd} 3^{-/}$mice were observed during their lifespan. In addition, van Gieson- and Alcian blue-stained connective tissue and cartilage of embryonal day (e)17, postnatal day (p)10, p20, 2-month, and 12-month-old WT and Smpd $^{--}$mice showed the highly disordered morphology of growth zones of long bones in Smpd3 $3^{--}$ mice. Age-dependent reduction of growth zones proceeded similarly in WT and Smpd $3^{-/-}$mice (Figure 6).

Control chondrocytes developed a dense ECM in WT mice, which is scarcely developed in Smpd $3^{--}$growth zones, visualized by anti-Col2A (Figure 7A). Cell 

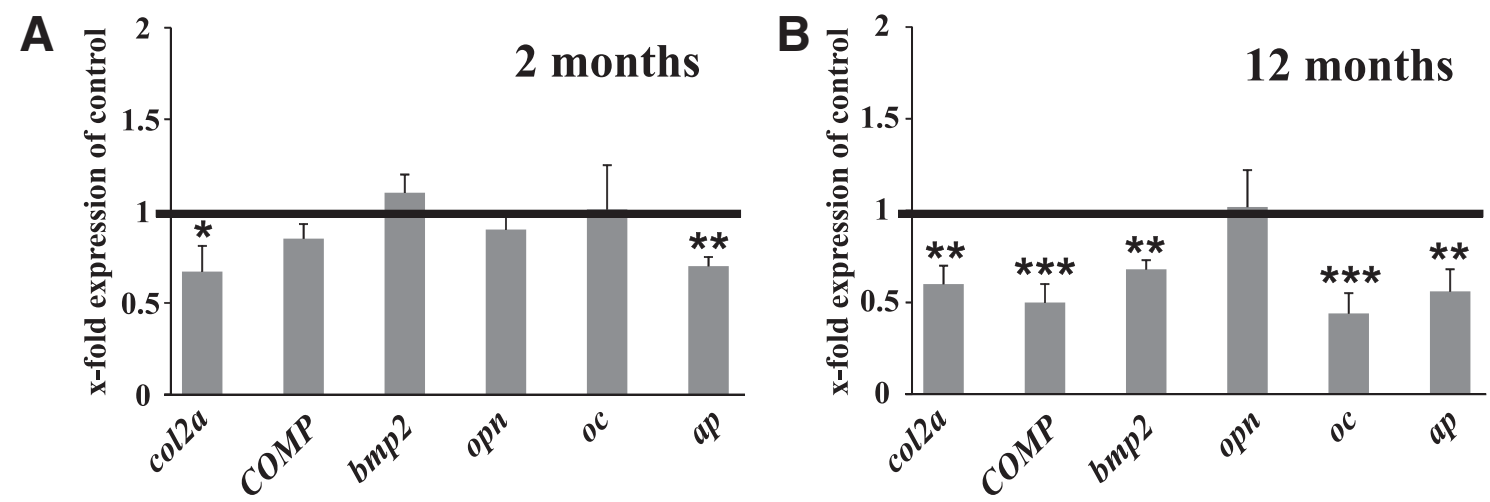

\section{2 months}
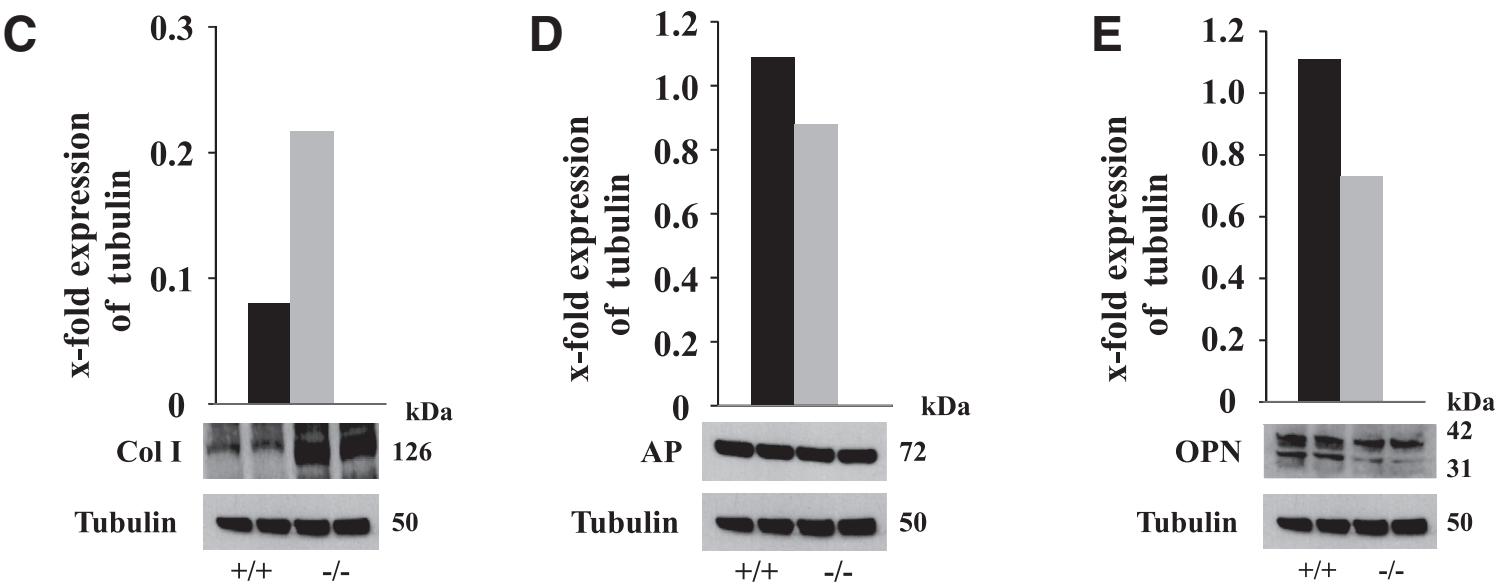

\section{2 months}
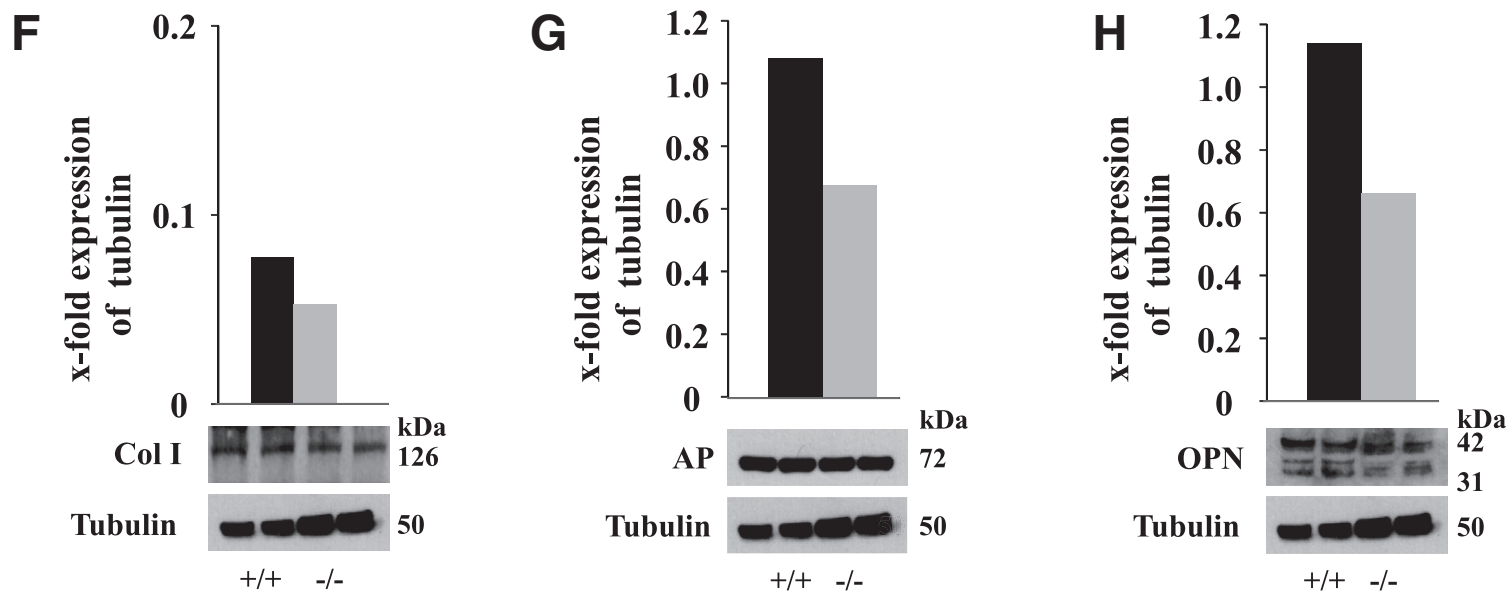

Figure 8 Transcriptional and translational expression of ossification-specific genes and proteins in long bone and osteoblasts in wild-type (WT) and Smpd $3^{\%}$ mice. A and B: Real-time PCR of complementary RNA of long bones of mice at (A) 2 and (B) 12 months of age, using primers of collagen 2a (col2a), cartilage oligomeric matrix protein (Comp), bone morphogenic protein2 (Bmp2), osteopontin (opn), osteocalcin (oc), and alkaline phosphatase (ap). Solid black line defines expression of control $(W T)=1$. C $-\mathbf{H}$ : Western blot analysis of protein lysates of osteoblasts in culture of mice at $2(\mathbf{C}-\mathbf{E})$ and $12(\mathbf{F}-\mathbf{H})$ months of age, using anti-collagen I (Col I), anti-alkaline phosphatase (AP), and anti-osteopontin (OPN) antibodies. $n=5$ per age and genotype. ${ }^{*} P<0.05,{ }^{* *} P<0.01$, and ${ }^{* * * P}<0.001$ versus WT.

proliferation was studied by quantifying the number of $\mathrm{Ki}$ 67-positive chondrocytes in the growth zone, which is reduced in humeri of $\operatorname{Smpd}^{--}: 90 \%$ of WT chondrocytes were Ki-67 positive (DAPI positive, 4262 ; Ki-67 positive, 3848 ), but only $70 \%$ in humeri of $\operatorname{Smpd} 3^{-/-}$siblings (DAPI positive, 3292; Ki-67 positive, 2310) (Figure 7B) in the e17 growth plate of $\mathrm{Smpd}^{--}$mice. These observations underline the results of our previous study in chondrocytes in culture. ${ }^{13}$ After embryonal and early postnatal ossification between e17 and p20, von Kossa staining indicated 

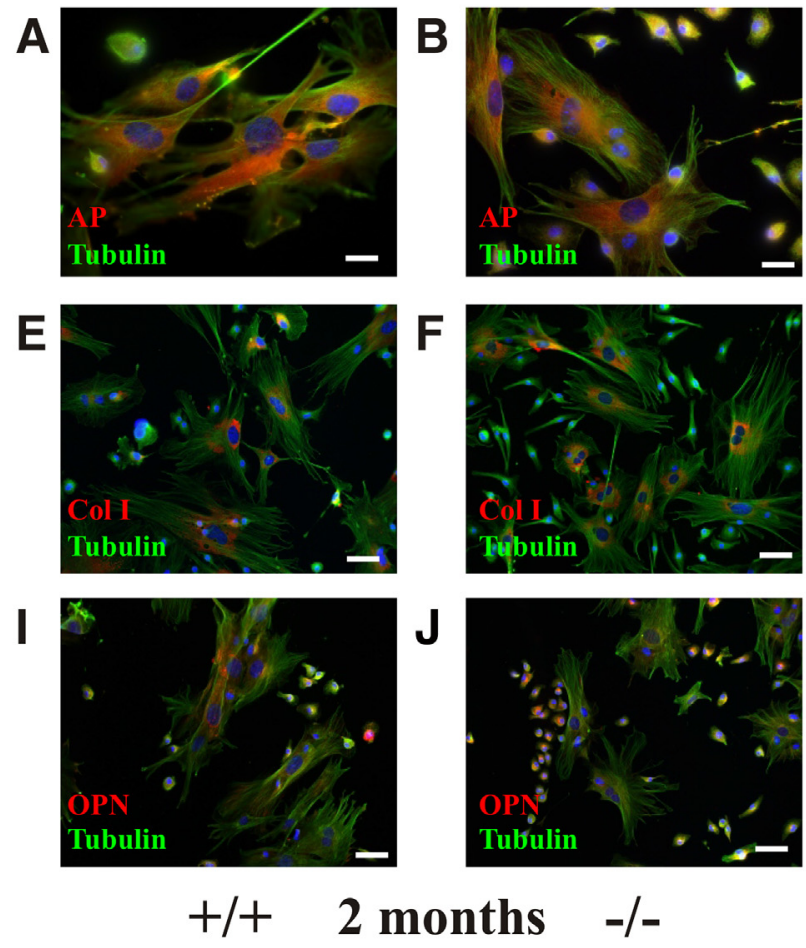
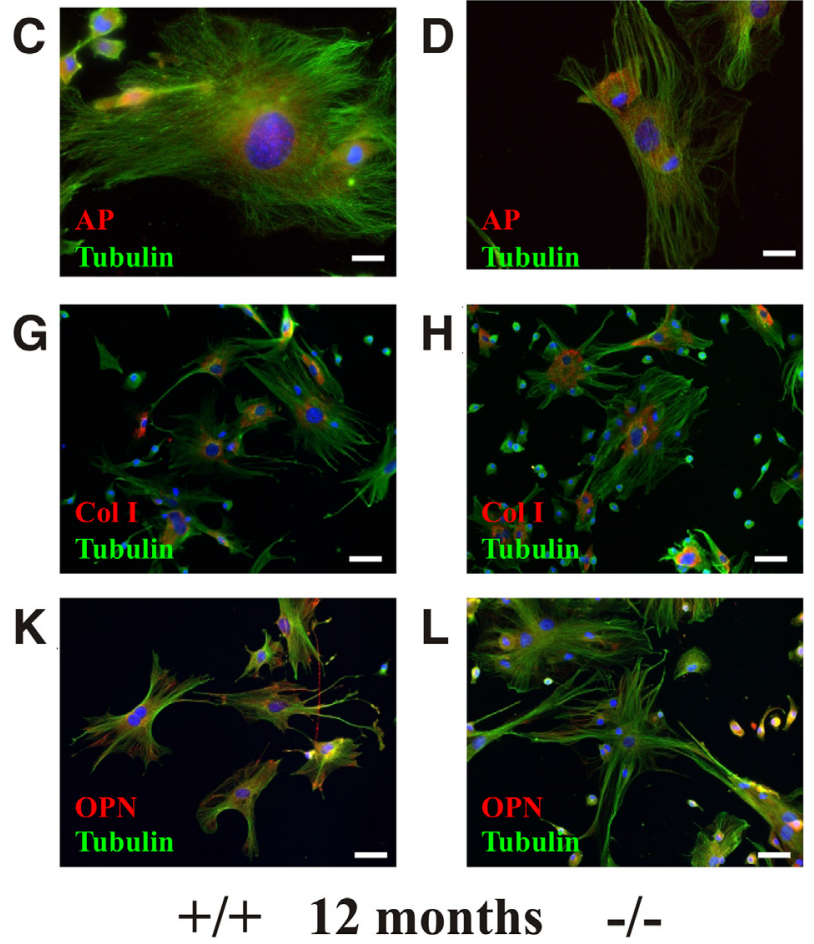

Figure 9 Immunohistochemistry of primary osteoblasts of 2- and 12-month-old wild-type WT and Smpd3\%- mice stained with antitubulin (fluorescein isothiocyanate) and anti-alkaline phosphatase (AP) (Cy3) (A-D), anti-collagen I (Col I) (Cy3) (E-H), and anti-osteopontin (OPN) antibody (Cy3) (I-L). Scale bars $=50 \mu \mathrm{m}$.

that the mineralization is slowed in long bones of e17 Smpd $3^{-/}$embryos (Figure 7D), but already similar to WT mice at p10 (Figure 7, C-F), but showed highly irregular ossification.

\section{Gene Expression in 0steogenesis of Long Bones of WT and Smpd3 $3^{-/}$Mice}

The expression of marker genes of osteogenesis was studied in total RNA of femora of juvenile (2 months) and adult (12 months) WT and Smpd $3^{-/}$mice by real-time PCR using specific primer pairs of bone morphogenic factor 2 , a member of the bone morphogenic family, that potently induces osteoblast differentiation, collagen 2a (col2a), and cartilage oligomeric matrix protein for monitoring matrix apposition and maturation, and of alkaline phosphatase (ap), osteocalcin (oc), and osteopontin (opn), key players in matrix mineralization and bone structure remodeling (Figure 8, A and B). Transcriptional activity was complemented by Western blot hybridization of protein lysates of osteoblasts in culture, isolated from femora of 2- and 12-month-old WT and $\operatorname{Smpd3^{--}}$ mice. Anti-collagen I (Col I) antibodies (Figure 8, C and F) were used to monitor ECM-protein apposition, anti-alkaline phosphatase (AP) antibodies for imaging mineralization (Figure 8, D and G), and anti-osteopontin (OPN) (Figure 8, E and H) antibodies were applied to monitor osteoclast activity.

These expression studies on the transcriptional and translational levels were complemented by comparative immunohistochemical analyses using antibodies of markers probing ECM apposition and longitudinal growth with anti-Col I, ossification mineralization with anti-alkaline phosphatase, and bone remodeling with anti-OPN antibodies (Figures 9 and 10).

Immunohistochemistry of primary osteoblasts indicated similar expression of alkaline phosphatase, Col I, and OPN during osteogenesis in 2- and 12-month-old WT and $\mathrm{Smpd}^{-/-}$mice (Figure 9).

Immunohistochemistry of long bones of 2-month-old WT and Smpd $3^{--}$mice showed high expression of Col I in the juvenile epiphysial and diaphysial growth zones (Figure 10A). Expression of alkaline phosphatase and OPN, indicators of mineralization and bone remodeling in the ossification zone, of 2- and 12-month-old WT and Smpd $3^{-1-}$ mice were nearly similar (Figure 10, B, C, D, E, and F).

\section{Discussion}

This study addressed the molecular basis of the role of neutral SMPD3 in sphingomyelin metabolism of chondrocytes in osteogenesis, longitudinal growth, and mineralization during the growth and growth-arrested phases using the unbiased genetic approach in the $\operatorname{Smpd} 3^{-/-}$mouse mutant. The Smpd $3^{--}$mutant develops a two-pronged novel form of juvenile dwarfism: systemic hypoplasia and tissuespecific chondrodysplasia and skeletal deformation. ${ }^{9}$ Growth retardation of the Smpd $3^{-/}$mouse mutant reflects a prolonged cell cycle and reduced proliferation rate during 

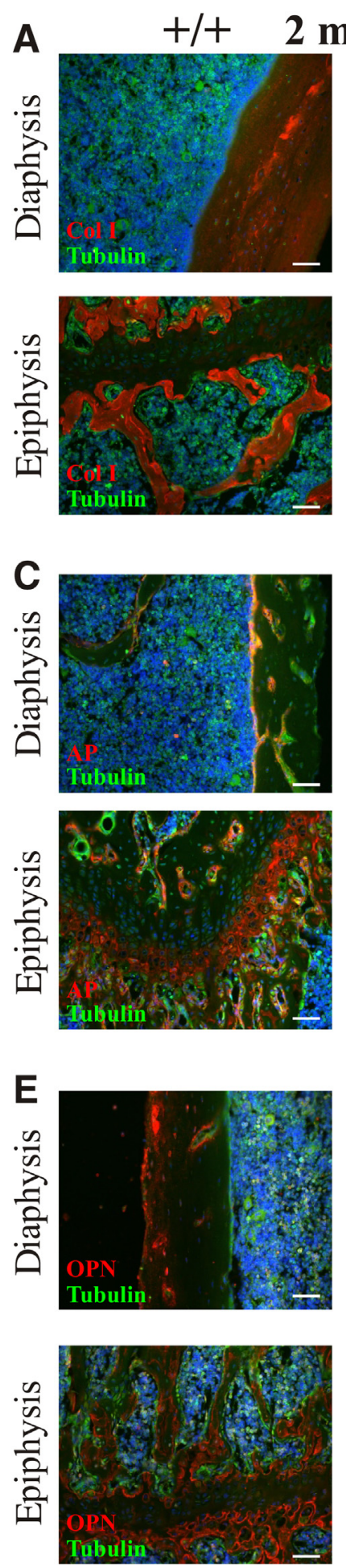
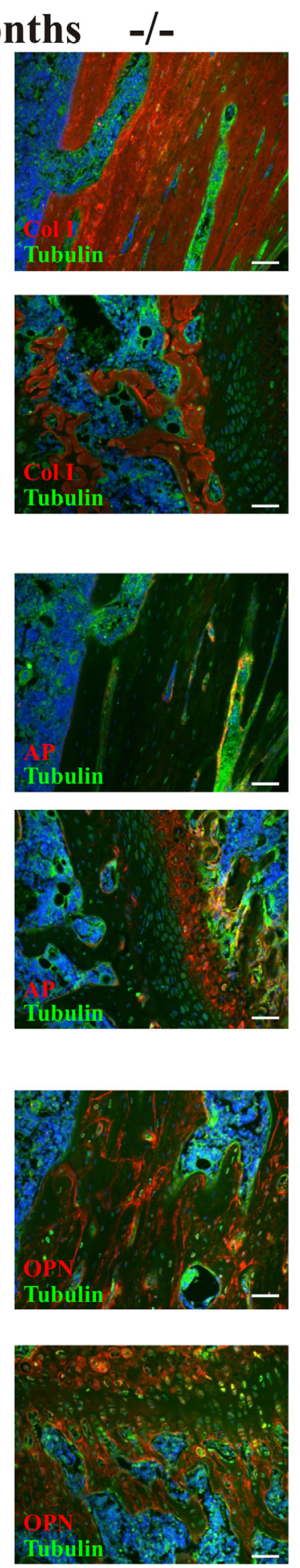

B
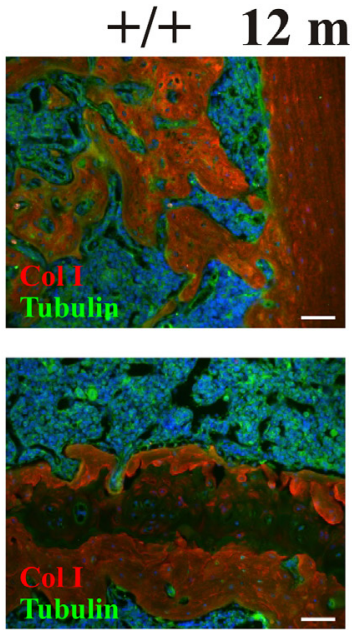

D
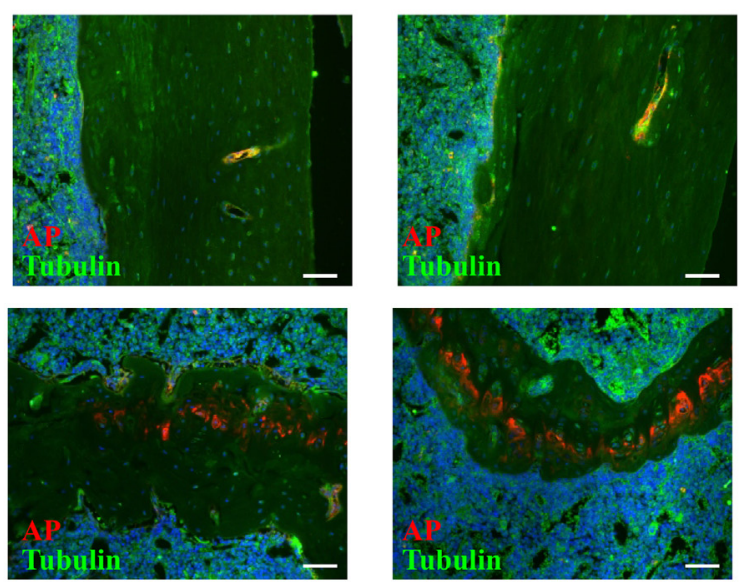

$\mathbf{F}$
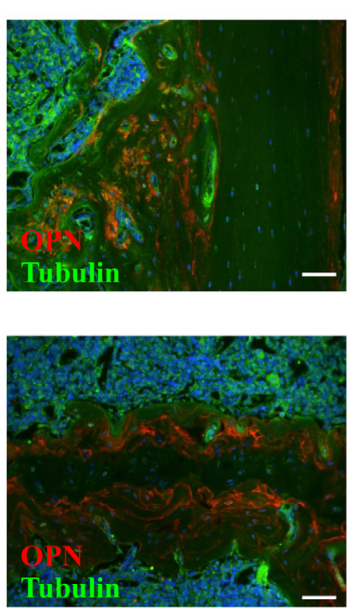
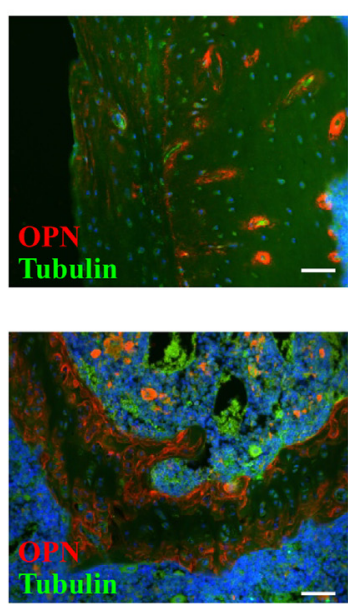

Figure 10 Immunohistochemistry of longitudinal sections of 2- and 12-month-old wild-type (WT) and Smpd3 $\%$ femora, stained with anti-tubulin (fluorescein isothiocyanate) and anti-collagen I (Col I) antibody (Cy3) (A and B), anti-alkaline phosphatase (AP) antibody (Cy3) (C and D), and anti-osteopontin (OPN) antibody (Cy3) (E and F). Scale bars $=50 \mu \mathrm{m}$.

the gestation and postnatal growth periods. The inhibition of Golgi vesicular protein transport as a unifying molecular link underlying SMPD3 deficiency was unveiled. Mechanistically, deficiency of Smpd3 expression does not affect the cell cycle as a growth gene directly. Slowed intracellular transport and secretion perturbs hypothalamic neurosecretion and causes hypothalamus-induced combined pituitary hormone deficiency. ${ }^{9}$ SMPD3 deficiency in chondrocytes of skeletal growth plates leads to intracellular accumulation of ECM proteins, collagens, matrilins, and cartilage oligomeric 


\section{$+/+$}
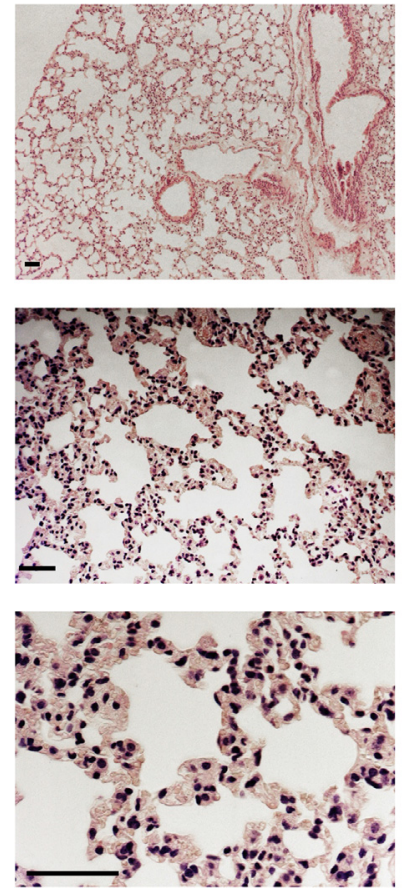

Figure 11 Normal alveolar structure of wild-type (WT) and Smpd3\% lung. Images of three magnifications of hematoxylin and eosin-stained sections of lung of adult (age, 4 months) wild-type (WT) and Smpd3\%mice. Scale bars $=50 \mu \mathrm{m}$.

matrix protein in the endoplasmic reticulum and Golgi stacks, disrupts proteostasis, induces unfolded protein response, ER stress, and premature apoptosis. ${ }^{13}$

The juvenile $\operatorname{Smpd} 3^{--}$mouse mutant shares hypoplasia of virtually all tissues and organs with that of the most common murine mutants developing a pituitary dwarf phenotype, the Snell mouse, Snell dwarf $\left(\mathrm{Pit}^{\mathrm{dw}} / \mathrm{Pit}^{1 \mathrm{dw}}\right)$, defective in pituitary-1 pit, the Ames dwarf (Prop $1{ }^{\mathrm{df}} / \mathrm{Prop} 1{ }^{\mathrm{df}}$, prophet of pit-1), and the little (lit) mouse mutant, Ghrhr ${ }^{\text {lit }} /$ Ghrhrit $^{\text {lit }}$, a missense mutation in the Ghrhr gene. ${ }^{20-26}$

Unlike the mutations of lysosomal acid sphingomyelinase, which causes sphingomyelin storage in the recombinant $S m p d 1^{-/-}$mice, ${ }^{6,7}$ equivalent to human Niemann-Pick disease type A, gene ablation of the neutral sphingomyelinase isozyme SMPD3, localized in the Golgi compartment, causes no sphingomyelin storage. $^{8}$

The loss of Smpd3 expression has no impact on the profiles and concentrations of phospholipid classes and their diacyl-glycerol species, as well as of sphingolipid classes sphingomyelin, ceramides, and glucosylceramide and their ceramide backbones in total lipid extracts of bones, and for comparison in brain of WT and Smpd $3^{--}$mice, the tissue with highest SMPD3 expression (Figures 1 and 2). These cumulative data and the previously described absence of a sphingolipidosis in the SMPD2-deficient mouse suggested cellular functions of neutral sphingomyelinases SMPD2 and SMPD3 in cellular compartments, the endoplasmic reticulum, and the Golgi-membrane stacks, respectively, other than the catabolic function of lysosomal acid sphingomyelinase SMPD1. ${ }^{6,8}$

Our recent studies elaborated the mechanism underlying the function of SMPD3 in the Golgi secretory pathway of chondrocytes providing ECM protein appositions for longitudinal growth in the skeletal growth zones for mineralization during the juvenile growth phase, lending bone architecture tensile strength. ${ }^{13}$ The role of SMPD3 in mineralization during osteogenesis is equivocally discussed. Our biochemical, immunohistochemical, and morphologic analyses combined with radiometry-densitometry, pQCT analysis, and high-resolution $\mu \mathrm{CT}$ measurements showed that SMPD3 function during osteogenesis is restricted to chondrocyte function, promoting the apposition of ECM proteins in the growth zones for longitudinal growth. The quantitative assessment of cortical and trabecular morphometry and density in juvenile and adult WT and $\operatorname{Smpd} 3^{-/}$female mice by these three methods unequivocally proved that mineralization is equal or even stronger in Smpd $3^{-/}$skeleton.

Radiography-densitometry and pQCT showed short femur length. $\mu \mathrm{CT}$ measurement showed unchanged trabecular and cortical total mineralized bone in WT and $S m p d 3^{--}$mice, indicating normal mineralization. Delayed secretion and irregular apposition of ECM proteins, ${ }^{13}$ of which $\mathrm{Col} \mathrm{I}$ and $\mathrm{Col} 2 \mathrm{a}$ are the dominant representatives, cause skeletal malformation and dysplastic joint pathology.

Morphologic analysis of growth zones of long bones by van Gieson staining of collagen structures of e17, p10, p20, 2-month-old, and 12-month-old WT and Smpd $3^{-1-}$ mice showed suppressed synthesis and secretion of ECM proteins (Figure 6A), and of cartilage glycosoaminoglycans specifically visualized by Alcian blue staining (Figure 6B). Col2A shows well-developed ECM in WT bone growth zones, whereas $\mathrm{Smpd}^{-/}$scarcely release the ECM marker Col2A (Figure 7B). After embryonal and early postnatal ossification, von Kossa staining indicated increasing mineralization in long bones, which proceeds to a similar extent and highly organized in WT, but irregularly structured in p20 Smpd $3^{--}$ mice. This provides an additional morphologic background for the chondrodysplastic phenotype.

These results are underscored by the transcriptional (realtime PCR) and translational (Western blot and immunohistochemistry) expression pattern of genes regulating ossification (Figure 8). The reduced transcriptional and translational expression of alkaline phosphatase in juvenile Smpd $3^{--}$mice indicates the delayed mineralization, allowing for bone and joint malformation owing to the lack of bone tensile strength. Osteopontin and osteocalcin are key players in bone remodeling. OPN stimulates osteoclasts to resorb bone and exert extracellular stress upon osteoblasts. ${ }^{27}$ Similarly, osteopontin, a $\gamma$-carboxyglutamic acid-containing peptide, which is produced exclusively by osteoblasts and secreted into the ECM, binds to $\mathrm{Ca}^{++}$and thus inhibits and delays mineralization. ${ }^{28}$ The reduced 
translational expression of OPN consequently might explain the enhanced mineralization in juvenile $S m p d 3^{-/}$mice. Although transcriptional expression in juvenile Smpd $3^{-/}$ mice is unaffected, expression in adult $S m p d 3^{-/-}$mice is reduced significantly.

Gene expression of marker proteins for ECM apposition in the growth zone, signaling activity of osteoblasts for longitudinal and ordered growth, and of osteoclasts in the ossification, was estimated by real-time PCR of complementary RNA of femora of WT and $S m p d 3^{-/-}$mice.

Production of Col I in Smpd3 $3^{-/}$osteoblasts during the growth phase exceeds that of WT osteoblasts. Col I is the major fibrillary protein in ECM of the growth plate, whereas Col2a with similar structure is down-regulated. Col I and $\mathrm{Col} 2 \mathrm{a}$ are the major collagens, which impose tensile strength on growing long bones.

A fro/fro (fragilitas ossium) mouse mutant was isolated from a pool of mutants generated by chemical mutagenesis of the genome of postmeiotic spermatids. ${ }^{14,15}$ The skeletal pathology of the fro mutation is characterized by hypomineralization, osteoporosis, limb deformation, multiple fractures of the long bone, and reduced mineralization in embryonic fro/fro osteoblasts in culture. The inactivation of the Smpd3 locus within a 980-kb deletion on chromosome 8 led to the conclusion that SMPD3 deficiency causes this phenotype, and the proposal that the fro/fro mouse mimics the recessive form of human osteogenesis imperfecta. ${ }^{29}$

Besides the genetic differences, this study discriminates the significantly different phenotypes of the fro/fro mutant and the well-defined Smpd3 ${ }^{-/-}$mutant. i) Gene targeting mutated the single Smpd3 locus on chromosome 8, different from the stochastic, chemical, mutagenized genome of the fro/fromutant. ii) No postnatal lethality of male and female Smpd3 ${ }^{-/}$ mice was observed, but rather a life span, prolonged by $22 \%$ compared with C57BL/6 control mice $(28.3 \pm 1.8$ versus $23.1 \pm 1.0$ months). ${ }^{13}$ The fro/fro mice show high perinatal lethality. ${ }^{15}$ Loss-of-function mutations at the single Smpd3 gene locus leads to a considerable (20\% to $25 \%$ ) extension of the life span. The Smpd3 null mouse therefore provides a novel model for the study of aging mechanisms in mammals. iii) Unlike the chronic respiratory obstructive lung pathology, similar to emphysema, which develops in the fro/fro mouse, ${ }^{16}$ both sexes of $S m p d 3^{--}$mice suffered no lung abnormalities during their life span. Histologic examination of lung sections of WT $(+/+)$ and $S m p d 3^{-/-}$mice, 15 months of age, showed normally structured alveolar texture, no inflated alveoli, empty sacs, and obstructive bronchioli, characterizing emphysema. No differences were seen in the continuity of the squamous alveolar cell linings of adjacent alveoli, sandwiched by morphologically inconspicuous interalveolar septa (Figure 11).

We conclude from our cumulative biochemical and cell biological results expanded with those elaborated by dualenergy X-ray absorptiometry, pQCT, and $\mu \mathrm{CT}$ used for quantifying bone structural parameters and bone mass architecture in WT and Smpd $3^{-/-}$littermates, that mineralization during ossification precedes unimpaired in the SMPD3-deficient mouse.

\section{Acknowledgment}

W.S., I.H., B.J., I.S.S., and A.N. performed the experiments; W.S., I.H., and A.N. analyzed the data; W.S. supervised the project, designed experiments, and wrote the manuscript.

\section{References}

1. Hannun YA, Obeid LM: The ceramide-centric universe of lipidmediated cell regulation: stress encounters of the lipid kind. J Biol Chem 2002, 277:25847-25850

2. Tomiuk S, Hofmann K, Nix M, Zumbansen M, Stoffel W: Cloned mammalian neutral sphingomyelinase: functions in sphingolipid signaling? Proc Natl Acad Sci U S A 1998, 95: $3638-3643$

3. Hofmann K, Tomiuk S, Wolff G, Stoffel W: Cloning and characterization of the mammalian brain-specific, $\mathrm{Mg} 2+$-dependent neutral sphingomyelinase. Proc Natl Acad Sci U S A 2000, 97: $5895-5900$

4. Wu BX, Rajagopalan V, Roddy PL, Clarke CJ, Hannun YA: Identification and characterization of murine mitochondria-associated neutral sphingomyelinase (MA-nSMase), the mammalian sphingomyelin phosphodiesterase 5. J Biol Chem 2010, 285:17993-18002

5. Hofmann K, Dixit VM: Reply to kolesnick and hannun, and perry and hannun. Trends Biochem Sci 1999, 24:227

6. Otterbach B, Stoffel W: Acid sphingomyelinase-deficient mice mimic the neurovisceral form of human lysosomal storage disease (NiemannPick disease). Cell 1995, 81:1053-1061

7. Horinouchi K, Erlich S, Perl DP, Ferlinz K, Bisgaier CL, Sandhoff K, Desnick RJ, Stewart CL, Schuchman EH: Acid sphingomyelinase deficient mice: a model of types A and B Niemann-Pick disease. Nat Genet 1995, 10:288-293

8. Zumbansen M, Stoffel W: Neutral sphingomyelinase 1 deficiency in the mouse causes no lipid storage disease. Mol Cell Biol 2002, 22: 3633-3638

9. Stoffel W, Jenke B, Block B, Zumbansen M, Koebke J: Neutral sphingomyelinase 2 (smpd3) in the control of postnatal growth and development. Proc Natl Acad Sci U S A 2005, 102:4554-4559

10. Hofmann K, Dixit VM: Ceramide in apoptosis-does it really matter? Trends Biochem Sci 1998, 23:374-377

11. Tomiuk S, Zumbansen M, Stoffel W: Characterization and subcellular localization of murine and human magnesium-dependent neutral sphingomyelinase. J Biol Chem 2000, 275:5710-5717

12. Zumbansen M, Stoffel W: Tumor necrosis factor alpha activates NFkappaB in acid sphingomyelinase-deficient mouse embryonic fibroblasts. J Biol Chem 1997, 272:10904-10909

13. Stoffel W, Hammels I, Jenke B, Binczek E, Schmidt-Soltau I, Brodesser S, Schauss A, Etich J, Heilig J, Zaucke F: Neutral sphingomyelinase (SMPD3) deficiency disrupts the Golgi secretory pathway and causes growth inhibition. Cell Death Dis 2016, 7:e2488

14. Guenet JL, Stanescu R, Maroteaux P, Stanescu V: Fragilitas ossium: a new autosomal recessive mutation in the mouse. J Hered 1981, 72: 440-441

15. Aubin I, Adams CP, Opsahl S, Septier D, Bishop CE, Auge N, Salvayre R, Negre-Salvayre A, Goldberg M, Guenet JL, Poirier C: A deletion in the gene encoding sphingomyelin phosphodiesterase 3 (Smpd3) results in osteogenesis and dentinogenesis imperfecta in the mouse. Nat Genet 2005, 37:803-805 
16. Poirier C, Berdyshev EV, Dimitropoulou C, Bogatcheva NV, Biddinger PW, Verin AD: Neutral sphingomyelinase 2 deficiency is associated with lung anomalies similar to emphysema. Mamm Genome 2012, 23:758-763

17. Committee for the Update of the Guide for the Care and Use of Laboratory Animals; National Research Council: Guide for the Care and Use of Laboratory Animals: Eighth Edition. Washington, DC, National Academies Press, 2011

18. Kilkenny C, Browne WJ, Cuthill IC, Emerson M, Altman DG: Improving bioscience research reporting: the ARRIVE guidelines for reporting animal research. J Pharmacol Pharmacother 2010, 2:94-99

19. Stauber M, Muller R: Micro-computed tomography: a method for the non-destructive evaluation of the three-dimensional structure of biological specimens. Methods Mol Biol 2008, 455:273-292

20. Butler AA, LeRoith D: Minireview: tissue-specific versus generalized gene targeting of the igfl and igflr genes and their roles in insulin-like growth factor physiology. Endocrinology 2001, 142: $1685-1688$

21. Daughaday WH, Rotwein P: Insulin-like growth factors I and II. Peptide, messenger ribonucleic acid and gene structures, serum, and tissue concentrations. Endocr Rev 1989, 10:68-91
22. Efstratiadis A: Genetics of mouse growth. Int J Dev Biol 1998, 42: 955-976

23. Le Roith D, Scavo L, Butler A: What is the role of circulating IGF-I? Trends Endocrinol Metab 2001, 12:48-52

24. Lupu F, Terwilliger JD, Lee K, Segre GV, Efstratiadis A: Roles of growth hormone and insulin-like growth factor 1 in mouse postnatal growth. Dev Biol 2001, 229:141-162

25. Liu JP, Baker J, Perkins AS, Robertson EJ, Efstratiadis A: Mice carrying null mutations of the genes encoding insulin-like growth factor I (Igf-1) and type 1 IGF receptor (Igf1r). Cell 1993, 75: $59-72$

26. Green H, Morikawa M, Nixon T: A dual effector theory of growthhormone action. Differentiation 1985, 29:195-198

27. Singh A, Gill G, Kaur H, Amhmed M, Jakhu H: Role of osteopontin in bone remodeling and orthodontic tooth movement: a review. Prog Orthod 2018, 19:18

28. Li J, Zhang H, Yang C, Li Y, Dai Z: An overview of osteocalcin progress. J Bone Miner Metab 2016, 34:367-379

29. Khavandgar Z, Poirier C, Clarke CJ, Li J, Wang N, McKee MD, Hannun YA, Murshed M: A cell-autonomous requirement for neutral sphingomyelinase 2 in bone mineralization. J Cell Biol 2011, 194:277-289 